\title{
Calcul aux éléments finis de l'écoulement aube à aube dans les pompes
}

\section{Finite-element calculation of blade-to-blade flow in pumps}

\author{
F. Martelli et G. Manfrida
}

Istituto di Energetica, Florence

\section{Introduction}

La méthodologie la plus courante pour l'étude de l'écoulement rotationnel de turbo-machines est basée sur la définition de 2 surfaces :

- la méridienne $S 2$ (souvent appelée "blade-like) - l'interpalaire $S 1$ (appelée en général "blade-toblade") $[1,2]$.

En particulier, l'étude sur la surface interpalaire permet :

a) de définir les profils de vitesse et de pression sur la surface de l'aube dans le but de calculs successifs de la couche limite ;

b) de calculer, grâce à une condition opportune au bord de sortie, la déviation donnée au flux par l'aube. Un tel phénomène, influenceé d'une façon plus ou moins importante, selon la géométrie de la machine, par la viscosité du fluide, peut en effet être convenablement simulé dans un calcul non visqueux.

Dans le cas où l'analyse du flux interpalaire est conduite par rapport d̀ la référence relative et la surface d'écoulement de type axoradiale, c'est à dire présentant une variation de rayon, les équations de flux sont typiquement non linéaires. On propose ici une solution itérative, grâce à la méthode des éléments finis, qui associe à la facilité de programmation la possibilité d'une discrétisation soignée pour des géométries particulières.

\section{Bases théoriques}

\subsection{Equations de l'écoulement}

L'équation de mouvement d'un fluide non visqueux peut s'écrire dans le système de référence relatif [3] :

$$
\bar{w} \times(\bar{\nabla} \times \bar{w})+2 \bar{w} \times \bar{\omega}=\bar{\nabla} I-T \bar{\nabla} S-\bar{D}
$$

où

$$
I=H-u c_{\theta}=h+\frac{w^{2}}{2}-\frac{u^{2}}{2} \text {. }
$$

En projetant l'équation (1) dans la direction $\eta$ localement orthogonale à la ligne de courant et avec l'hypothèse de symétrie axiale de la surface d'écoulement, on obtient :

$$
\begin{aligned}
w\left[\frac{1}{r} \frac{\partial w_{m}}{\partial \theta}-\frac{\partial w_{\theta}}{\partial m}\right]=\frac{d I}{d \eta} & -T \frac{\partial s}{\partial \eta} \\
& +\sin \alpha\left[2 \omega+\frac{w_{\theta}}{r}\right] w
\end{aligned}
$$

où l'angle $\alpha$ définit localement l'inclinaison de la surface de l'écoulement par rapport à la direction axiale (fig. 1).

La résolution de l'équation de continuité implique l'existence d'une fonction de courant ainsi définie :

$$
\begin{aligned}
& \frac{\partial \psi}{\partial m}=-b \rho w_{\theta} / \dot{m} \\
& \frac{\partial \psi}{\partial \theta}=r b \rho w_{m} / \dot{m}
\end{aligned}
$$

où $b$ est la hauteur locale du canal en direction normale par rapport à la surface $S 1$ (fig. 1).

Dans le cas d'écoulement incompressible, on obtient l'équation de mouvement telle que :

$$
\frac{\partial}{\partial \theta}\left[\frac{1}{r^{2}} \frac{\partial \psi}{\partial \theta} \frac{b_{\infty}}{b}\right]+\frac{\partial}{\partial m}\left[\frac{\partial \psi}{\partial m} \frac{b_{\infty}}{b}\right]=\frac{\sin \alpha}{q}\left[\frac{w_{\theta}}{r}+2 \omega\right]
$$
avec $q=m\left(\rho_{\infty} b_{\infty}\right)$,
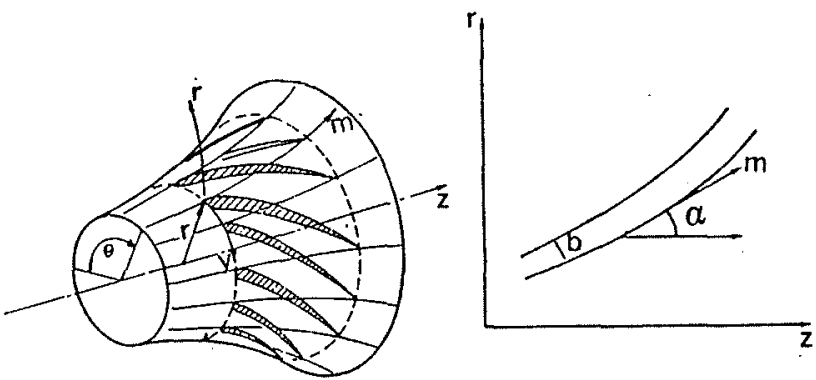

Figure 1 - Définition de la surface axisymetrique aube à aube $S 1$.

LA HOUILLE BLANCHE/N ${ }^{\circ} 7 / 8-1982$ 
et où sont omis pour briéveté les termes de variation de rothalpie et d'entropie.

En se reportant à la figure 2 , les conditions à la limite pour l'équation (5) sont résumées dans le tableau $\mathbf{I}$.

\begin{tabular}{|c|c|c|}
\hline \multicolumn{3}{|c|}{ Tableau I } \\
\hline Limite & Nom & Condition \\
\hline 1 & Section d'entrée A.m costante & $w_{\theta}=w_{\theta 1}$ \\
\hline 2 & Paroi solide, cốté en dépression & $\psi=0$ \\
\hline 3 & Section de sortie A.m costante & $w_{\theta}=w_{\theta} 3$ \\
\hline 4 & Paroi solide, côté en pression & $\psi=1$ \\
\hline \multirow[t]{3}{*}{$5 A, 5 B$} & Limite de périodicité & $\psi_{B}=\psi_{A}+1$ \\
\hline & & $\partial \psi \quad \partial \psi$ \\
\hline & & $\partial n_{B} \cdot \partial n_{A}$ \\
\hline
\end{tabular}

La condition à la limite sur le côté 3 implique l'assignation de l'angle de sortie. Celui-ci peut être calculé grâce à des corrélations expérimentales, ou en posant une condition sur les valeurs de vitesse (ou de pression) dans le voisinage du bord de sortie.

L'equation (5) peut être récrite sous forme variationelle si le $2^{\mathrm{e}}$ terme est considéré en fonction des seules coordonnées $m-\theta$. Sous cette forme, on a :

$$
\begin{aligned}
\pi=\int_{\Omega}\left\{\frac{1}{2}\left[\frac{b_{\infty}}{b r^{2}}\left(\frac{\partial \psi}{\partial \theta}\right)^{2}+\frac{b_{\infty}}{b}\left(\frac{\partial \psi}{\partial m}\right)^{2}\right]\right. \\
\left.+\psi \frac{\sin \alpha}{q}\left[\frac{w_{\theta}}{r}+2 \omega\right]\right\} d \Omega+\int_{\Gamma} \frac{w_{t}}{q} \psi d \Gamma
\end{aligned}
$$

en même temps que la condition $\delta \pi=0$

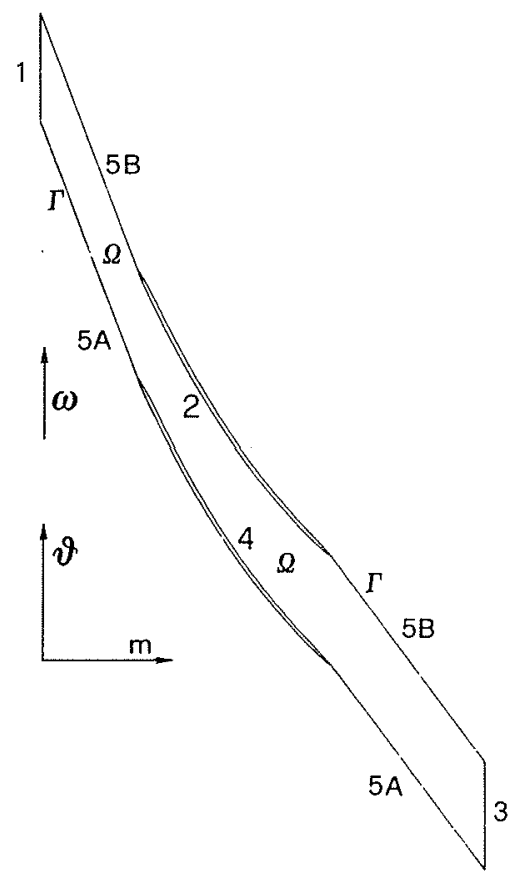

Figure 2-Domaine de calcul et ses contours
Cette dernière condition, correspondant à la stationnarité du fonctionnel $\pi$, comme on peut le vérifier en calculant la variation et en intégrant par parties équivaut à l'équation (5).

L'équation (6) peut aussi s'obtenir grâce à l'application de la méthode des résidus pesés, dans la forme de Galerkin [3], à partir de l'équation 5 avec les conditions à la limite associées.

Dans la formulation variationelle on distingue :

- des conditions à la limite de type fort, qui sont satisfaites de façon exacte et correspondent aux valeurs assignées à la fonction $\psi$ (dans ce cas, les conditions sur les limites 2 et 4 de la figure 2 , ainsi que la $\psi_{B}=$ $\psi_{A}+1$ sur la limite 5 )

- et des conditions de type faible, correspondant aux valeurs assignées à la dérivée normale de la fonction $\psi$, dont la précision dépend de la discrétisation qui est effectuée du fonctionnel et qui sont seulement satisfaites approximativement (comme les conditions sur les côtés 1 et 3 de la figure 2 , ainsi que la $\left.\frac{\partial \psi}{\partial n}\right|_{B}=$ $\left.\frac{\partial \psi}{\partial n}\right|_{A}$ sur la limite 5). Sur la base de cette classification, la condition générale à la limite 5 peut être considérée de type mixte.

\subsection{Discrétisation du fonctionnel grâce aux éléments finis}

Le domaine d'intégration du fonctionnel, indiqué sur la figure 2, peut être discrétisé grâce à la théorie des éléments finis [4]. Dans ce cas, il est préférable de maintenir la notation de l'équation (6), et de discrétiser le domaine avec les variables $m-\theta$, sans introduire de variable linéaire du type $y=r \theta$. Un tel choix s'impose dans le cas où l'analyse doit être étendue à des machines de genre axoradial ou radial, avec lesquelles on peut avoir de fortes variations de rayon même à l'intérieur d'un seul élément : de telle façon il est possible de respecter avec simplicité la condition physique d'imperméabilité de la paroi.

En introduisant des fonctions de forme $N_{i}$, n'importe quelle variable (par exemple $\psi$ ) à l'intérieur de l'élément peut s'écrire, en utilisant les éléments triangulaires à 3 nouds :

$$
\psi=\Sigma_{i} N_{i}(\theta, m) \cdot \psi_{i}
$$

avec $i=1,2,3$

La substitution de l'expression ( 7 ) dans la relation (6) permet l'évaluation du fonctionnel $\pi$ en tant que fonction des valeurs nodales $\psi, \psi_{i}$

La condition $\delta \pi=0$, correspondant à la différentiation par rapport à un vecteur générique de $\left(\psi_{i}\right)$ nodal, porte à la construction d'un système linéaire, du genre :

$$
\left[k_{i j}\right]\left\{\psi_{i}\right\}=\left\{g_{i}\right\}
$$

avec $i=1, \ldots \ldots, p$

et $j=1, \ldots \ldots, p$

dans lequel $p$ est le nombre total de nœuds. 
En outre :

$$
\begin{gathered}
k_{i j}=\Sigma^{e} \int_{\Omega_{e}}\left[\frac{\partial N_{i}}{\partial \theta} \frac{\partial N_{j}}{\partial \theta} \frac{b_{\infty}}{b r^{2}}+\frac{\partial N_{i}}{\partial m} \frac{\partial N_{j}}{\partial m} \frac{b_{\infty}}{b}\right] d \Omega^{\prime} \\
\left\{g_{i}\right\}=\left\{g_{i}^{\prime}\right\}+\left\{g_{i}^{\prime \prime}\right\} \\
g_{i}^{\prime}=\Sigma^{e} \int_{\Omega_{e}} \sin \alpha \sum_{1}^{3} \frac{b_{\infty}}{b r_{j}} w_{\theta} N_{j} N_{i} d \Omega_{e} \\
-\Sigma_{e} \int_{\Omega_{e}} \frac{2 \omega \sin \alpha}{q} N_{i} d \Omega_{e}+ \\
g_{i}^{\prime \prime}=-\frac{1}{q} \Sigma_{e} \int_{\Gamma_{5}^{e}} w_{t} N_{i} d \Gamma^{e} \int_{\Gamma_{1,3}^{e}} w_{t} N_{i} d \Gamma^{E}
\end{gathered}
$$

où $e$ est l'indice de l'élément générique.

Le terme $g_{i}^{\prime \prime}$ représente la contribution au deuxième membre des intégrales de limite sur les frontières de périodicité ; on peut les évaluer seulement à travers la connaissance des composantes locales de vitesse tangentes à ces limites, qui sont à priori inconnues. De tels termes inconnus peuvent être éliminés grâce à une transformation appropriée de la matrice $k_{i j}$, basée sur la combinaison linéaire des lignes et sur la propriété $\psi_{5 B}=\psi_{5 A}+1$. Le système (8) devient donc

$$
\left[k_{i j}^{\prime}\right]\left\{\psi_{i}\right\}=\left\{g^{\prime}\right\}
$$

$$
\text { avec } \begin{aligned}
i & =1, \ldots \ldots, p \\
j & =1, \ldots \ldots, p
\end{aligned}
$$

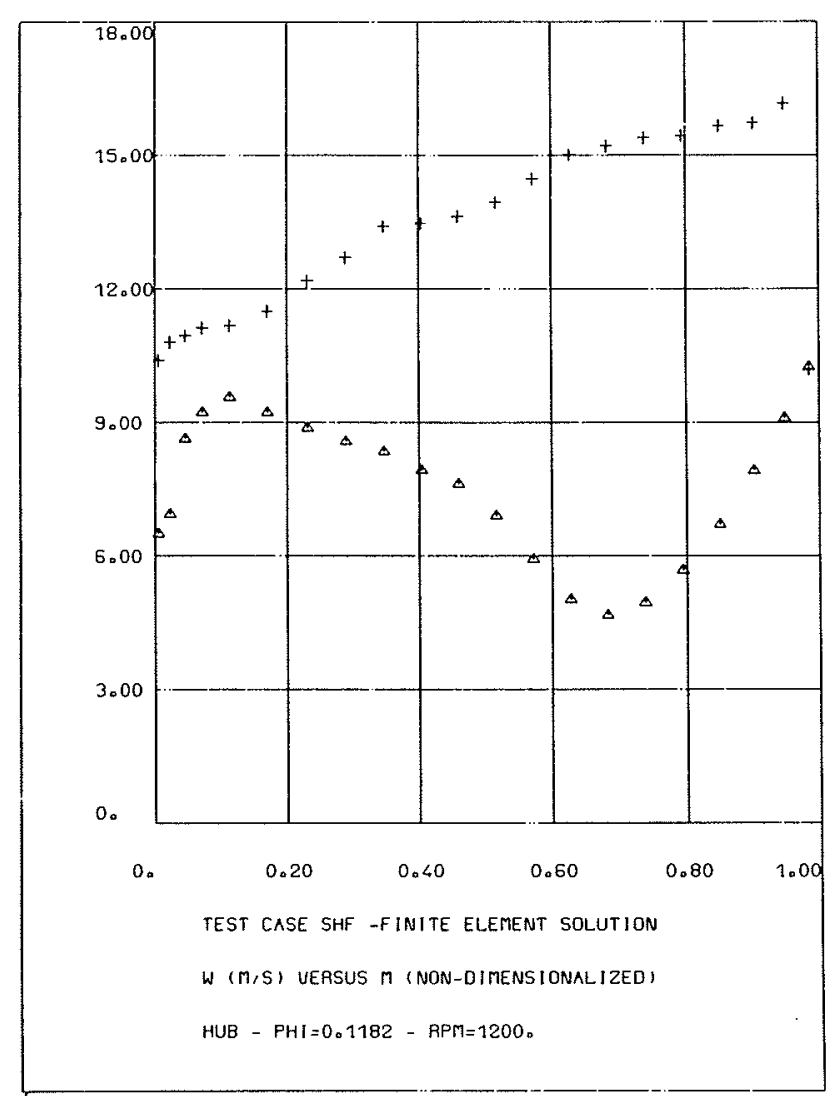

Figure 3 - Roue SHF, ceinture de roue : vitesse relative calculée.

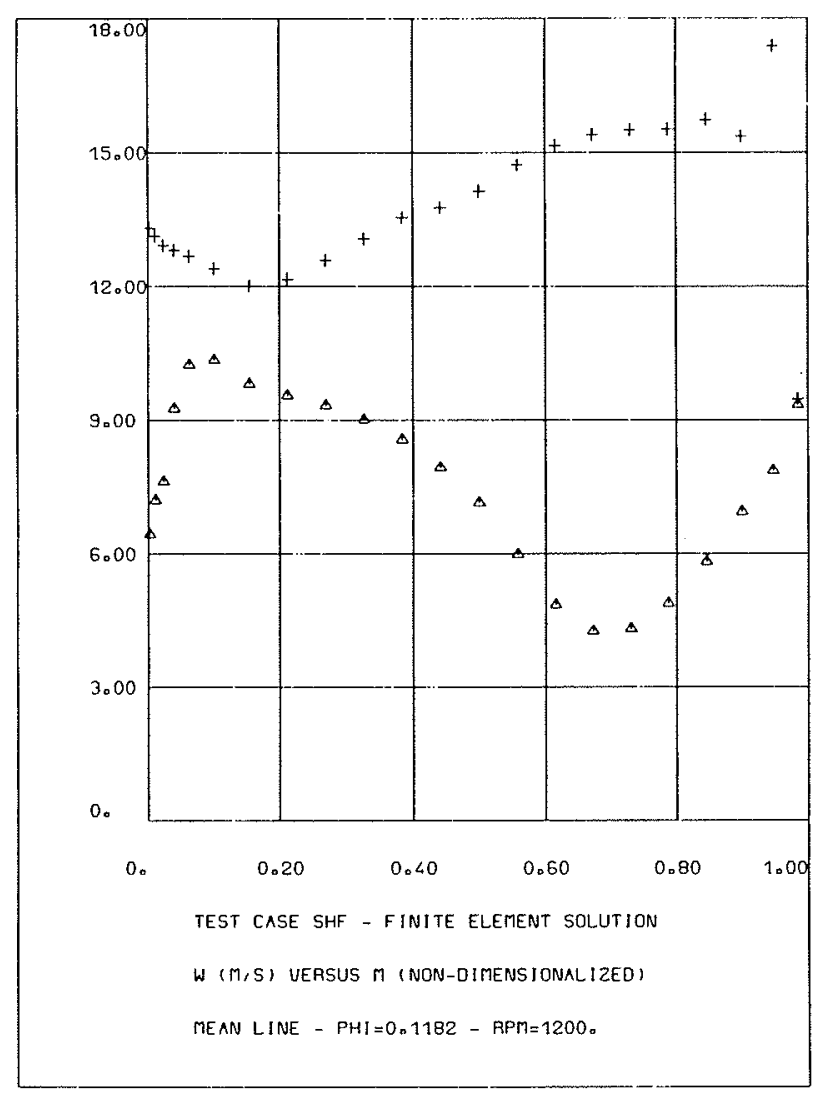

Figure 4 - Roue SHF, surface moyenne : vitesse relative calculée.

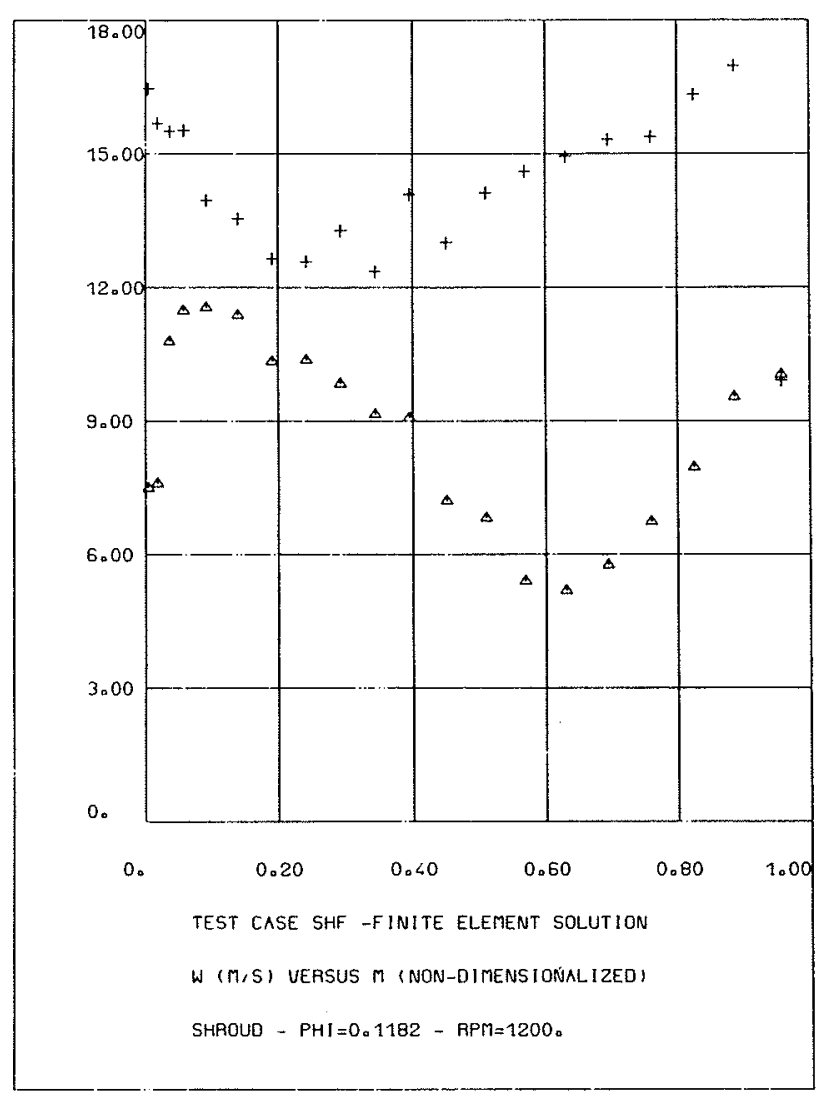

Figure 5 - Roue SHF, plafond de roue : vitesse relative calculée. 
Des méthodes itératives de relâchement local permettent de résoudre le système $\left(8^{\prime}\right)$, avec l'avantage d'un traitement aisé des conditions de périodicité et d'une formulation compacte du code de calcul.

Dans l'équation (9), la variation de $\frac{b_{\infty}}{b r^{2}}, \frac{b_{\infty}}{b}$ à l'intérieur de l'élément est, en toute rigueur, non linéaire. Pour éviter de recourir à des intégrations numériques on peut admettre d'approcher cette loi par une loi linéaire donnée par les fonctions de forme, comme il a déjà été fait implicitement dans le deuxième membre pour les termes $\frac{b_{\infty}}{b r}$.

Il est évident du fait de l'équation (11) que, pour la connaissance du deuxième membre, la fonction $\psi$, dont dépend $w_{\theta}$, doit être connue dans le domaine considéré. On propose donc un algorithme itératif, dans lequel la solution à chaque intégration est utilisée pour l'évaluation du deuxième membre dans les calculs successifs jusqu'à la convergence.

\section{Synthèse des résultats}

\subsection{Roue $S H F$}

La géométrie de cette roue constitue un test numérique-expérimental proposé par la $S^{\text {té }}$ Hydrotechnique de France [5].

On a effectué des calculs pour cette géométrie sur les trois surfaces de révolution correspondantes à la ceinture, au moyeu et au plafond de la roue. L'épaisseur de la surface d'écoulement en direction normale à la sur-

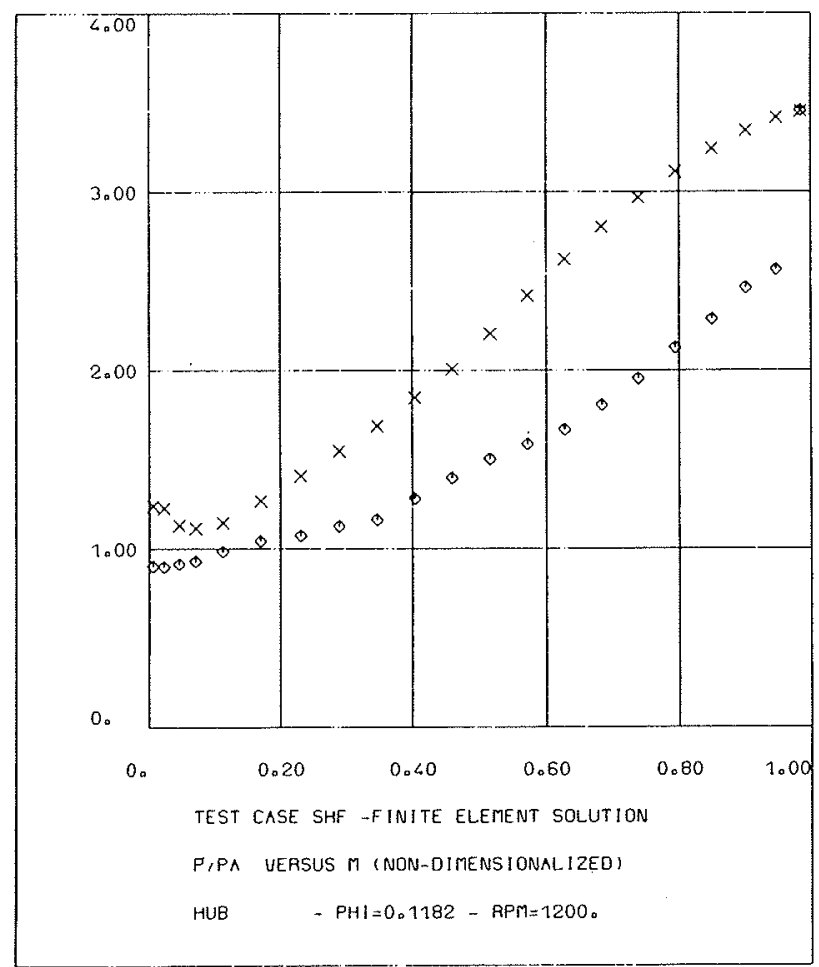

Figure 6 - Roue SHF, ceinture de roue : rapport de pression calculé.

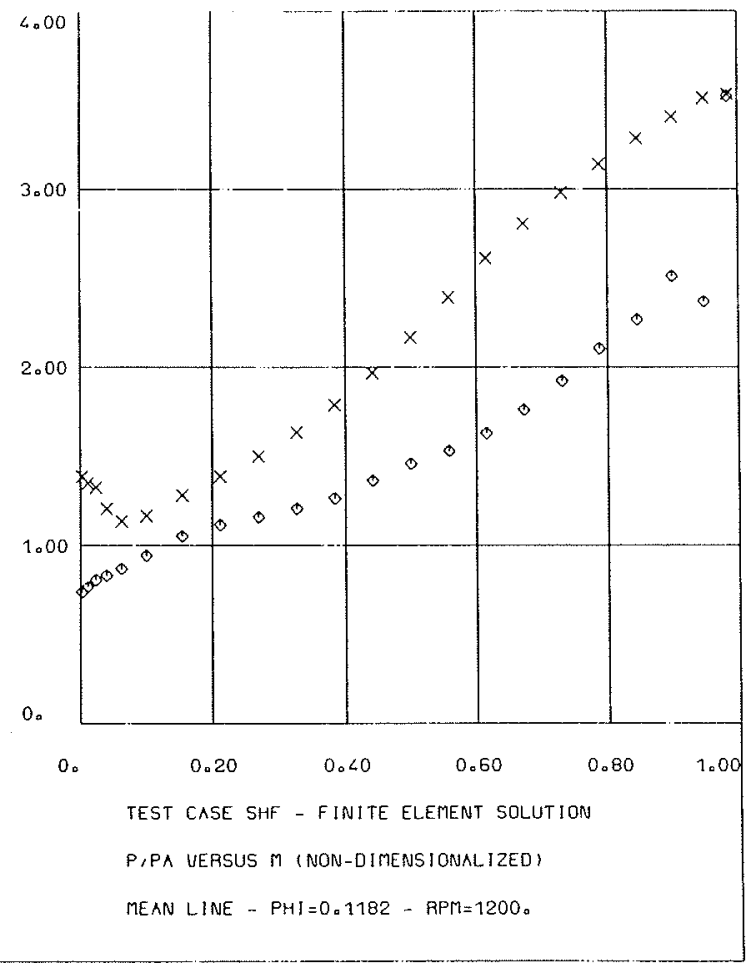

Figure 7 - Roue SHF, surface moyenne : rapport de pression calculé.

face même a êté déterminée au moyen d'un calcul méridien [5].

La discrétisation du domaine a demandé un nombre variable de noeuds compris entre 1100 et 1250 .

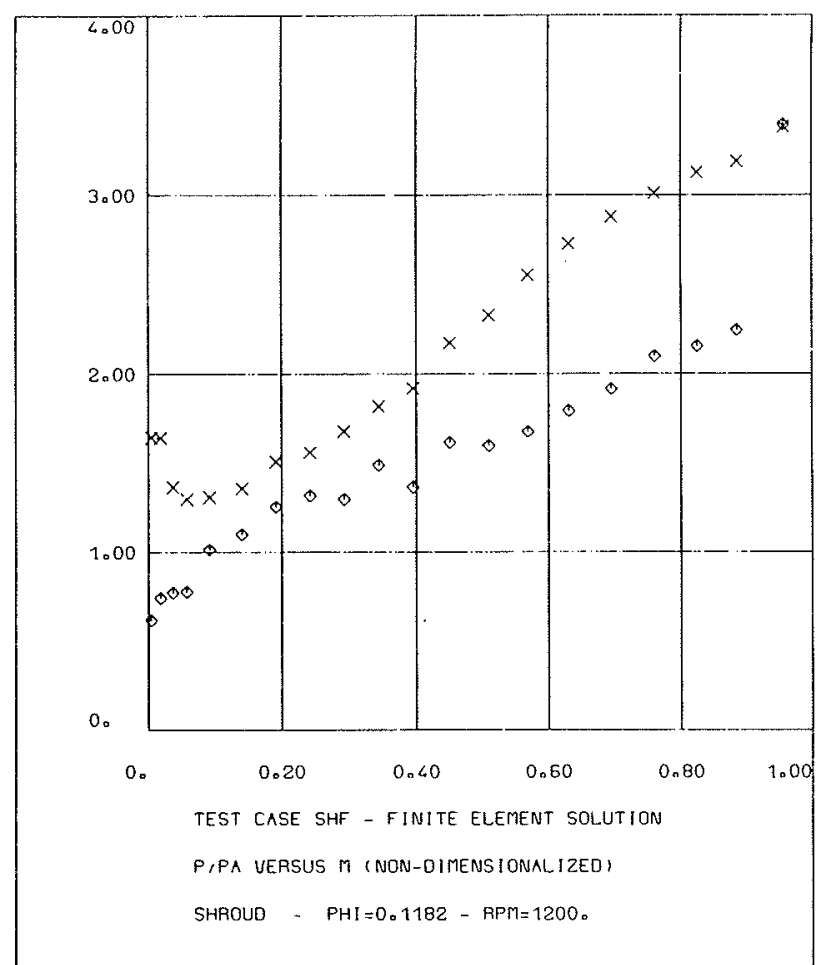

Figure 8 - Roue SHF, plafond de roue : rapport de pression calculé. 


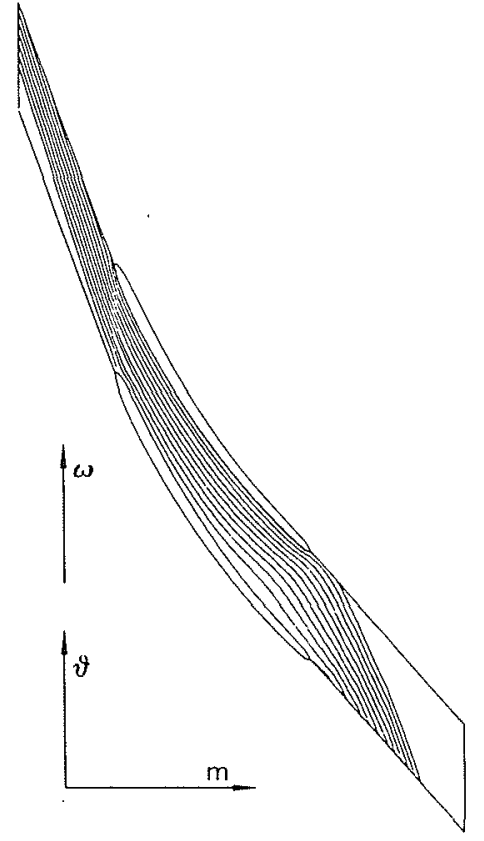

Figure 9 - Roue SHF, lignes équicourant, surface $m-\partial$

Les profils de vitesse sur l'aube sont mentionnés sur les figures 3-4-5 pour les trois surfaces de calcul. On remarque notamment l'effet de l'incidence positive à l'entrée $\left(6^{\circ}\right.$ au plafond, $8^{\circ}$ à la surface moyenne, $10^{\circ}$ à la ceinture de la roue) pour le débit de calcul $(1121 / \mathrm{s}$, $\phi_{2}=0.118$ ).

L'angle de sortie relatif se situe entre $14^{\circ}$ et $15^{\circ}$ par rapport à la direction tangentielle.

Les profils de pression, non-dimensionalisés par rapport à la pression atmosphérique, sont réprésentés sur les figures 6-7-8. Il y a lieu de remarquer que la condition à la sortie de la roue permet de tendre vers le même rapport $p_{2} / p_{a}$ pour les trois surfaces $\left(p_{2} / p_{a}=\right.$ 3.45).

Pour la surface moyenne, on a établit une carte des lignes d'écoulement, c'est-à-dire des lignes ayant la même

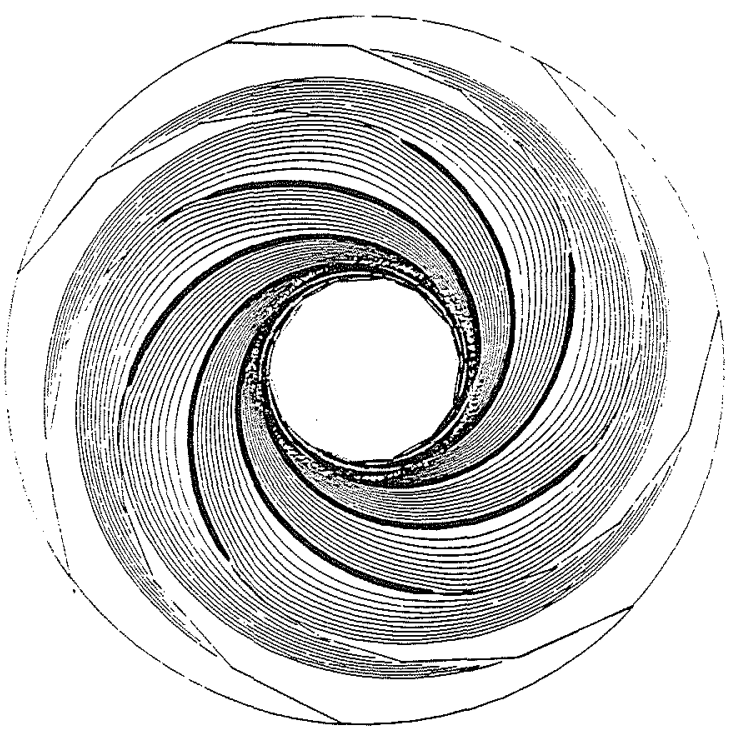

Figure 10 - Roue SHF, plot polaire des lignes equicourant. valeur de la fonction de courant. Ces lignes sont représentées sur le plan de calcul $m-\theta$ par la figure 9, tandis que la figure 10 représente une vue d'ensemble des mêmes lignes pour la roue complète en projection polaire $(\nu-\nu \partial)$, projection capable de rendre l'apparence physique de l'écoulement dans une roue centrifuge. On remarque également sur les figures 9-10, l'effet de l'incidence à l'entrée, l'attraction des lignes de courant sur la surface en dépression de l'aube, et le phénomène de "slip" à la sortie de la roue.

\subsection{Roue "Watanabe" [6]}

Les résultats de la méthode de calcul ont été vérifiẻs sur des données expérimentales pour une roue centrifuge avec écoulement dans l'air, ce qui représente le "test-case" d'un récent symposium international [6]. Cette roue est complètement instrumentée pour le relevé des pressions sur la surface des aubes en rotation, et constitue en cela un intéressant banc d'essai pour tout calcul d'écoulement. Des résultats théoriques sont aussi repérables en littérature.

La roue a été examinée en conditions de projet $\left(\phi_{2}=0.4\right)$ et hors $-\operatorname{projet}\left(\phi_{2}=0.3\right.$ et 0.5$)$.

Les résultats pour $\dot{\varphi}_{2}=0.5$ ne sont pas significatifs du point de vue expérimental, du fait de la présence d'une séparation très large (incidence négative sur les aubes). La comparaison entre le calcul proposé, les résultats expérimentaux et les calculs d'autres auteurs est représentée par les figures 11-12 (respectivement pour $\dot{\varphi}_{2}=0.4$ et $\phi_{2}=0.3$ ).

Sur cette simple géométrie, caractérisée par un "inducer" et par des aubes à direction purement radiale $\left(\beta_{2}=90^{\circ}\right)$, les calculs théoriques donnent des résultats comparables. Les différences entre les résultats théoriques et l'expérience sont comparables à une séparation

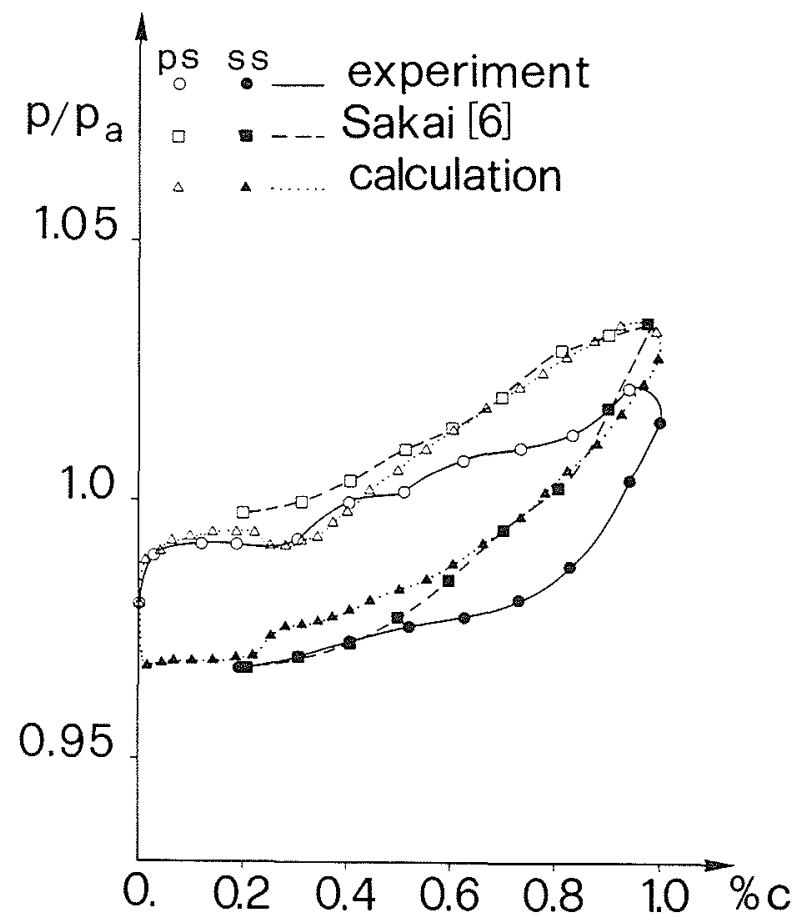

Figure 11 - Roue Watanabe, rapport de pression, $\phi_{2}=0.4$. 


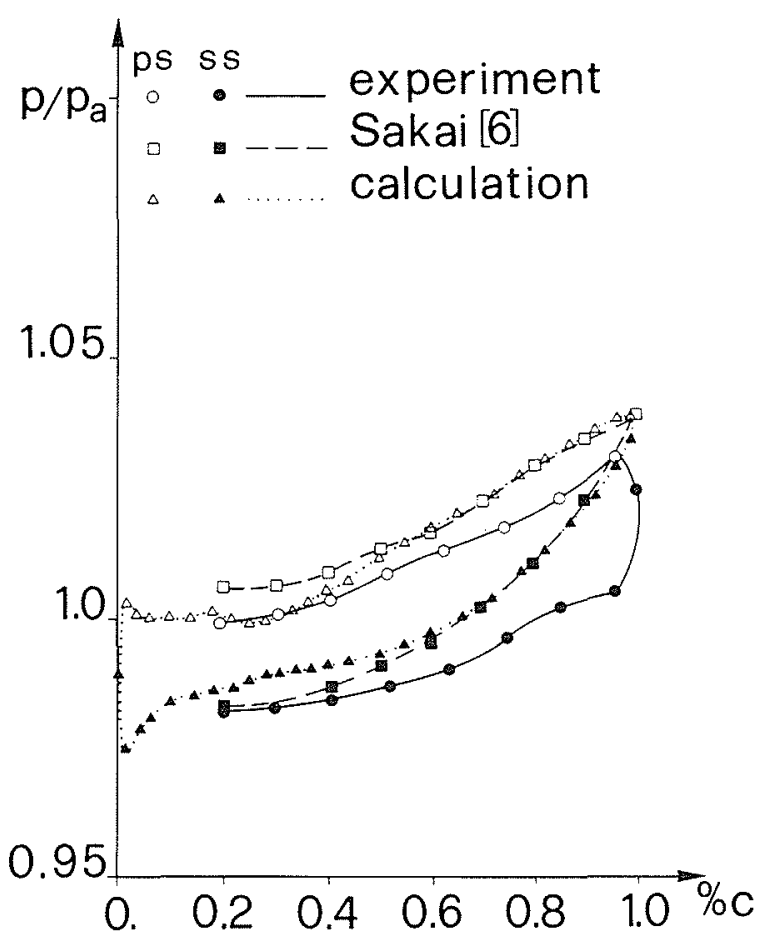

Figure 12 - Roue Watanabe, rapport de pression, $\phi_{2}=0.3$.

visqueuse qui a été observée par les expérimentateurs et est due notamment à une mauvaise conformation du canal méridien.

\section{Conclusions}

Du point de vue de l'utilisateur, le modèle de calcul proposé s'est révélé économique et d'utilisation facile : le temps de calcul est limité à $20 \mathrm{~s}$ sur un CDC 7600 , avec un coût de $15+20 \mathrm{FF}$ par opération. La prépara- tion des données est facilitée par la présence généralisée de sous-programmes d'interpolation "spline" qui permettent, dans la plupart des géométries, de limiter notamment le nombre des données nécessaires pour la définition de la surface d'écoulement.

Parmi les perspectives de développement caractéristiques de ce modèle de calcul, on peut citer :

1) la possibilité d'obtenir la condition sur le bord de sortie directement et non plus par itération du calcul 2) le développement d'un modèle de séparation du type jet-wake, pour tenir compte de la modification de la géométrie de la surface d'écoulement à suite de la séparation.

3) le traitement complet des gradients de rothalpie, d'entropie et des forces dissipatives

4) la transformation du modèle en un calcul de projet, c'est-à-dire capable de définir la géométrie de l'aubage à partir des profils donnés de vitesse que l'on veut obtenir.

\section{Bibliographie}

[1] WU C.H. - A General Theory of the Two-Dimesional Flow in Subsonic and Supersonic Turbomachines of Axial, Radial and Mixed - Flow Type, NACATN 2604.

[2] BOSMAN C., MARSH H. - An Improved Method for Calculating the Flow in Turbomachines, Including a Consistent Loss Model, Journal Mechanical Engineering Science, Vol. 16 n. 1 (1974).

[3] MARTELLI F. - In tema di analisi fludodinamica in schiere di pale con gli elementi finiti, Congresso ATI 1980.

[4] ZIENKIEWICZ O.C. - The Finite Element Method Mc Graw-Hill Book Co 1975 .

[5] CARNEVALE E., GIUSTI S., ANGELO G. - Analyse Théorique-expérimentale de l'écoulement réel dans les pompes axoradiales. $121^{\mathrm{e}}$ Session SHF - Paris 17-18 Novembre 1982 .

[6] MIZUKI S., ARIGA T., WATANABE I. - Investigation Concerning the Blade Loading of Centrifugal Impellers. ASME Paper n. 74 - GT-143. 


\section{Discussion}

Président: J. DODU

M. le Président. - Merci, M. MARTELLI, de votre communication et d'avoir bien voulu employer notre langue.

M. CORNIGLION. - J'ai personnellement très apprécié l'exposé de M. MARTELLI puisqu'il constitue avec ce qui a été présenté par M. CARNEVALE une méthode de calcul quasitridimensionnelle de l'écoulement d'un fluide compressible dans une turbomachine. Ce travail est analogue à celui présenté lors de l'ouverture de cette session par M. EREMEEF et concerne le calcul quasi $3 \mathrm{D}$ décrit déjà en 1951 par Pr. C.H.WU. Le principe étant de décomposer l'écoulement $3 \mathrm{D}$ en deux écoulements $2 \mathrm{D}$ couplés. Tout l'art de l'ingénieur revient à effectuer le couplage le plus adapté possible afin qu'il représente au mieux l'écoulement réel. Et c'est pour celà qu'un groupe de la S.H.F. s'occupe de la comparaison des méthodes numériques d'une part et de la comparaison théorie-expérience d'autre part.

Pour en revenir plus concrètement à votre tex te, je remarque que dans l'équation générale des turbomachines (équation $n^{\circ} 1$ de votre texte), vous faites intervenir une variation d'entropie comme pour les fluides compressibles. Ne serait-il pas préférable de parler de perte de pression totale qui est à mon sens, pour des fluides incompressibles, beau coup plus significative?

M. MARTELLI. - Je crois que c'est seulement une question d'écriture. Il y a une correspondance entre le gradient d'entropie et les variations de pression totales dans l'écoulement, on peut utiliser l'une ou l'autre écriture: c'est seulement une question de valeurs numériques des différents paramètres.

M. CORNIGLION. - La variation d'entropie pour un fluide incompressible, pour notre cas, est faible.

M. MARTELLI. - Les variations d'entropie, pour des pertes de pressions totales très grandes, sont en effet petites c'està-dire que l'on pourrait commettre une imprécision sar la variation d'Entropie. Pourtant les constructeurs des pompes préférent introduire les pertes en terme de pression totale.

\section{CORNTGLION. - Je le pense.}

M.CARNEVALE. - Nous avons introduit un modèle de perte et, dans nos équations, il apparait un gradient d'entropie; mais dans les calculs que nous avons présenté hier, on a pu voir à la fin que nous avions introduit un coefficient de perte qui est le rapport entre l'augmentation de pression totalc dans l'évolution réelle et l'augmentation de pression totale dans l'évolution isoentropique. Celà nous permet d'utiliser les résultats d'un premier calcul isoentropique pour l'évaluation du gradient d'entropie, mais nous avons aussi mis en corrélation ce gradient avec la perte de pression totale.

\section{Abstract \\ Finite-element calculation of blade-to-blade flow in pumps}

Inviscid flow numerical calculations offer a valid tool for the analysis and design of turbomachines: usually, the coupling of two sets of bidimensional calculations, a first one on the meridional surface (S2) and a second one on the blade-toblade (S1) surface, is used to obtain a quasi-three-dimensional simulation of the flow in the turbomachine. The second type of calculation ( $\mathrm{S} 1$ surface) is particularly important, as it gives important informations of the velocity and pressure field inside the bladed channel, and it can lead, through the use of an adequate closure condition, to a satisfactory calculation of the outlet angle (which is actually determined by the true, viscous flow).

The numerical difficulties which are encountered in such a calculation range from the generation of a satisfactory mesh to the treatment of periodic flow boundaries and to the nonlinearity of the flow equations: this last is particularly relevant if the flow surface is three-dimensional, i.e. in mixed or radial flow impellers of the type common in pumps.
The proposed numerical analysis is based on the solution of the flow equations on an axisimmetric S1 surface in the coordinates $m$ (meridional abscissa) $\theta$ (tangential angular abscissa), thus obtaining a relevant uniformity of approach with respect to the most widespread codes (Katsanis etc.). The discretization of the flow surface is done by means of threenode Finite Elements, after a quasi-variational reformulation of the problem. The input is simplified by a generalized use of spline extrapolation techniques, which allow satisfactory discretizations to be obtained starting by a limited set of impeller geometric data.

The results of the calculation are presented for two testcases: a first one, proposed by SHF, on which experimental data will be available within a short time, and a second one, whose results in terms of theoretical calculations and experiments are available in the literature. 